\title{
Curvaton mechanism and its implications to sneutrino cosmology
}

\author{
Takeo Moroi $^{\mathrm{a}}$ \\ ${ }^{a}$ Department of Physics, Tohoku University, Sendai 980-8578, Japan \\ I describe basic features of the curvaton scenario where the primordial fluctuation of a late-decaying scalar field, \\ called "curvaton," becomes the dominant source of the cosmic density fluctuations. I also discuss its implications \\ to sneutrino cosmology.
}

\section{Introduction}

In the study of the evolution of the universe, it is important to understand the origin of the cosmic density fluctuations. The most conventional scenario is inflation [1] where quantum fluctuation of the inflaton field during the inflation becomes the origin of the cosmic density fluctuations. From the particle-physics point of view, it is desirable to construct a model of inflation which is testable by collider (or other laboratory) experiments; if it is possible, we can study the mechanism of the cosmological density fluctuations with collider experiments. It is, however, difficult to construct such a testable model of inflation since the requirements on the inflaton potential is very stringent.\#1

Recently, a new mechanism of generating the cosmic density fluctuations has been attracting many attentions, where a late-decaying scalar condensation provides the dominant source of the cosmic density fluctuations. In this scenario, dominant part of the cosmic density fluctuations originate from the primordial fluctuation of a new scalar field, called "curvaton," which is different from the inflaton field 345]. Even though, in a large class of the curvaton scenario, inflation is assumed as a solution to the horizon and flatness problems as well as to generate the primordial fluctuation of the curvaton field, constraints on the inflaton potential can be relaxed in the

\footnotetext{
\#1 Within the minimal supersymmetric standard model, however, it may be possible to use some of the scalar quarks and Higgs bosons as the inflaton. For detail, see 2 .
}

curvaton scenario. In addition, since the requirements on the curvaton is not so stringent, it is possible to use (some of the) well-motivated particles as the curvaton; for example, scalar fields in the minimal supersymmetric standard model (MSSM) may play the role of the curvaton. In such a case, it may be possible to study the properties of the fields responsible for the structure formation by collider experiments. (For detailed studies of the curvaton scenario, see $[\underline{6}$.)

Here, I will review the curvaton scenario and discuss some of its implications to particle cosmology. Organization of the rest of this article is as follows. In Section 2 I first discuss the thermal history we consider. Then, in Section 3 I explain the curvaton scenario. The cosmic microwave background (CMB) angular power spectrum generated in the curvaton scenario is studied in Section 4 In Section 5 implications to the sneutrino cosmology is discussed. Section [ is devoted for conclusions and discussion.

\section{Thermal history}

I first discuss the basic scenario. In the curvaton scenario, there are two scalar fields which play important roles; one is the inflation $\chi$ and the other is the curvaton field $\phi . \# 2$ The most important features of the curvaton scenario do not depend on the details of the inflation. Thus, I do not assume any specific form of the inflaton

\#2 I assume the inflation to solve the horizon and flatness problems. The curvaton mechanism may be implemented in the pre-big-bang 7 and the ekpyrotic [8] scenarios. In this article, however, I will not discuss those cases. 
potential. On the contrary, resultant density fluctuations depend on the potential of the curvaton field. Here, for simplicity, I adopt the simplest form of the curvaton potential, i.e., the parabolic one:

$V(\phi)=\frac{1}{2} m_{\phi}^{2} \phi^{2}$,

where $m_{\phi}$ is smaller than the expansion rate of the universe during the inflation $H_{\text {inf }}$. In addition, the curvaton field is assumed to have nonvanishing initial amplitude $\phi_{\text {init }}$.

In the scenario, the universe starts with the inflationary epoch and, after the inflation, the universe is reheated by the decay of the inflaton. Then, the universe is dominated by the radiation (which I call $\gamma_{\chi}$ ). I call the radiation-dominated epoch filled with radiation generated from the decay products of the inflaton as the first radiation dominated epoch, or RD1 epoch, since, in the curvaton scenario, there are two radiationdominated epochs. Denoting the decay rate of the inflaton as $\Gamma_{\chi}$, reheating temperature after the inflation is given by $\# 3$

$T_{\mathrm{R} 1} \sim \sqrt{M_{*} \Gamma_{\chi}}$,

where $M_{*}$ is the reduced Planck scale. In the early stage of the RD1 epoch, slow-roll condition is satisfied for the curvaton field. As the universe expands, however, the curvaton field starts to oscillate. When $\Gamma_{\phi} \lesssim H \lesssim m_{\phi}$ (with $\Gamma_{\phi}$ being the decay rate of $\phi$ ), energy density of $\phi$ behaves as that of non-relativistic matter. Then, the energy density of the curvaton $\rho_{\phi}$ is proportional to $a^{-3}$ while the energy density of $\gamma_{\chi}$ is proportional to $a^{-4}$, where $a$ is the scale factor. Thus, as the universe expands, curvaton dominates the universe (if the lifetime of the curvaton is long enough). I call this epoch as curvaton-dominated epoch, or $\phi \mathrm{D}$ epoch. The curvaton field decays when the expansion rate becomes comparable to the decay rate of the curvaton. The reheating temperature due to the curvaton decay is given by

$T_{\mathrm{R} 2} \sim \sqrt{M_{*} \Gamma_{\phi}}$.

\#3 In the following discussions, I neglect $O(1)$ coefficients which are not important.

\section{Curvaton mechanism: evolutions of the fluctuations}

In the curvaton scenario, $\phi$ also acquires the primordial fluctuation during the inflation. Denoting the curvaton field (with comoving momentum coordinate $\vec{x}$ ) as $\# 4$

$\phi(t, \vec{x})=\phi(t)+\delta \phi(t, \vec{x})$,

let us consider the two-point correlation function of the fluctuation:

$$
\begin{aligned}
& \langle 0|\delta \phi(t, \vec{x}) \delta \phi(t, \vec{y})| 0\rangle= \\
& \quad \int \frac{d k}{k} \frac{d \Omega_{\vec{k}}}{4 \pi}|\delta \phi(t, \vec{k})|^{2} e^{i \vec{k}(\vec{x}-\vec{y})} .
\end{aligned}
$$

Then, the Fourier amplitude generated during the inflation is given by

$\delta \phi(t, \vec{k})=\left(\frac{k}{2 a H_{\mathrm{inf}}}\right)^{m_{\phi}^{2} / 3 H_{\mathrm{inf}}^{2}}\left[\frac{H_{\mathrm{inf}}}{2 \pi}\right]_{k=a H_{\mathrm{inf}}}$,

where the subscript " $k=a H_{\mathrm{inf}}$ " implies that the quantity is evaluated at the time of the horizonexit during the inflation. As I discuss below, the primordial fluctuation of $\phi$ given in Eq. (3.3) becomes the origin of the cosmic density fluctuations in the curvaton scenario.

Now, we are at the position to discuss the evolutions of the cosmological density fluctuations generated from $\delta \phi_{\text {init }}$. For this purpose, I use the Newtonian gauge where the line element is described with the metric perturbations $\Psi$ and $\Phi$ as $\# 5$

$$
\begin{aligned}
d s^{2} & =-(1+2 \Psi) d t^{2}+a^{2}(1+2 \Phi) d \vec{x}^{2} \\
& =a^{2}\left[-(1+2 \Psi) d \tau^{2}+(1+2 \Phi) d \vec{x}^{2}\right],
\end{aligned}
$$

where $\tau$ is the conformal time. In addition, the variable $\delta_{X}$ is defined as

$\delta_{X} \equiv \delta \rho_{X} / \rho_{X}$

where the subscript $X$ denotes the individual components (like radiation, cold dark matter

\#4 Here, I use the same notation for the zero-mode and for the total amplitude since there should be no confusion.

\#5 Here, I use the notation and convention of [9] unless otherwise mentioned. 
(CDM), baryon, and so on) and $\delta \rho_{X}$ is the fluctuation of the energy density of $X$.

Substituting Eq. (3.4) into the Einstein equation, we obtain the generalized Poisson equation for $\Phi$ :

$k^{2} \Phi=\frac{1}{2 M_{*}^{2}} a^{2} \rho_{\text {tot }}\left[\delta_{\text {tot }}+\frac{3 \mathcal{H}}{k}\left(1+\omega_{\text {tot }}\right) V_{\text {tot }}\right]$.

Here, "tot" denotes the total matter and the variable $V_{X}$ denotes the velocity perturbation of the component $X$. Furthermore, $\omega_{\text {tot }} \equiv \rho_{\text {tot }} / p_{\text {tot }}$ is the equation-of-state parameter for the total matter, and

$\mathcal{H} \equiv \frac{1}{a} \frac{d a}{d \tau}$.

I consider the situation where the temperature of the universe is so high that the momentumexchange of relativistic particles are efficient enough. In this case, perturbation of the radiation becomes locally isotropic and the anisotropic stress perturbation vanishes, resulting in $\Psi+\Phi=$ 0 .

When the perturbation of the radiation becomes locally isotropic, the equations for the density and velocity perturbations of the radiation are given by

$\delta_{\gamma}^{\prime}=-\frac{4}{3} k V_{\gamma}-4 \Phi^{\prime}$

$V_{\gamma}^{\prime}=\frac{1}{4} k \delta_{\gamma}+k \Psi$

where the "prime" denotes the derivative with respect to the conformal time $\tau$. In addition, if a very weakly interacting non-relativistic component exists, its perturbations obey the following equations:

$\delta_{m}^{\prime}=-k V_{m}-3 \Phi^{\prime}$,

$V_{m}^{\prime}=-\mathcal{H} V_{m}+k \Psi$,

where the subscript " $m$ " is for non-relativistic matters. Notice that, when a scalar field is oscillating, the equation-of-state parameter of the scalar condensation vanishes and hence the density and velocity perturbations of the scalar field also obey Eqs. (3.10) and (3.11).
In the curvaton scenario, it is assumed that the primordial fluctuation $\delta \phi_{\text {init }}$ becomes the dominant source of the cosmic density fluctuations. Thus, hereafter, I will discuss the density fluctuations generated from $\delta \phi_{\text {init }}$. When $\Gamma_{\phi} \lesssim H \lesssim m_{\phi}$, the situation is like the case with isocurvature fluctuation in non-relativistic matter component [10. In the curvaton case, as in the conventional isocurvature case, it is convenient to define the (primordial) entropy fluctuation between $\phi$ (and its decay products) and the photon from the inflaton $\gamma_{\chi}$ :

$S_{\phi \chi} \equiv\left[\delta_{\phi}-\frac{3}{4} \delta_{\gamma_{\chi}}\right]_{\mathrm{RD} 1}$.

Then, the cosmological density fluctuations are parameterized by using $S_{\phi \chi}$.

In order to discuss the evolutions of the fluctuations, it is important to know the equation-ofstate parameters of individual components in the universe. If all the components behave as the relativistic or the non-relativistic matter, evolutions of the perturbations are described by Eqs. (3.8) - (3.11). In this case, it is convenient to distinguish the photon (or any other components) from the decay product of $\phi$ from that from the inflaton field, which I call $\gamma_{\phi}$ and $\gamma_{\chi}$, respectively. ${ }^{\# 6}$ In order to consider $\delta_{\gamma_{\phi}}$ in the RD2 epoch, we can neglect $\gamma_{\chi}$ since the CMB radiation at this epoch is dominantly from the $\phi$ field. Then, we find that, in the RD2 epoch, $\Psi$ and $\delta_{\gamma_{\phi}}$ become constant while $V_{\gamma_{\phi}}=O(k \tau)$ up to higher order corrections. Indeed, combining Eq. (3.6) with Eqs. (3.8) and (3.9), and using $\delta_{\text {tot }}=\delta_{\gamma_{\phi}}$ and $V_{\text {tot }}=V_{\gamma_{\phi}}$, we obtain $V_{\gamma_{\phi}}^{(\delta \phi)}=-\frac{1}{2} k \tau \Psi_{\mathrm{RD} 2}^{(\delta \phi)}$ and

$\delta_{\gamma_{\phi}}^{(\delta \phi)}=-2 \Psi_{\mathrm{RD} 2}^{(\delta \phi)}$,

where $\Psi_{\mathrm{RD} 2}^{(\delta \phi)}$ is the metric perturbation induced by the primordial fluctuation of the amplitude of

\#6 In fact, these photons are mixed each other and they cannot be defined separately. In other words, their velocity perturbations should be the same since they form a single fluid. Even so, the following arguments are unchanged as far as we consider the leading terms in the density perturbations since the velocity perturbation is at most $O(k \tau)$. In the following discussion, $\gamma_{\phi}$ and $\gamma_{\chi}$ should be understood as representatives of the components which are and are not generated from the decay product of $\phi$, respectively. 
$\phi$. (In the following, the superscript " $(\delta \phi)$ " is for perturbations generated from the primordial fluctuation of $\phi$.) As I mentioned, $\Psi_{\mathrm{RD} 2}^{(\delta \phi)}$ is constant up to a correction of $O\left(k^{2} \tau^{2}\right)$.

Behavior of $\delta_{\gamma_{\chi}}^{(\delta \phi)}$ is also easily understood. In discussing the effects of the primordial fluctuation of $\phi$, we neglect the initial fluctuation of the inflaton field and hence $\delta_{\gamma_{\chi}}^{(\delta \phi)} \rightarrow 0$ in the deep RD1 epoch. In addition, from Eqs. (3.8) and (3.9), $V_{\gamma_{\chi}}^{(\delta \phi)}$ becomes higher order in $k \tau$ than $\delta_{\gamma_{\chi}}^{(\delta \phi)}$ and $\Psi^{(\delta \phi)}$. Thus, we obtain

$\delta_{\gamma_{\chi}}^{(\delta \phi)}=4 \Psi^{(\delta \phi)}$.

The above relation holds in the $\mathrm{RD} 1, \phi \mathrm{D}$, and RD2 epochs up to corrections of $O\left(k^{2} \tau^{2}\right)$.

$\left[\Psi^{(\delta \phi)}\right]_{\mathrm{RD} 2}$ can be related to $S_{\phi \chi}$. Using the fact that the entropy fluctuation is a conserved quantity for superhorizon mode, the following relation holds:

$$
\begin{aligned}
S_{\phi \chi}^{(\delta \phi)} & =\left[\frac{3}{4} \delta_{\gamma_{\phi}}^{(\delta \phi)}-\frac{3}{4} \delta_{\gamma_{\chi}}^{(\delta \phi)}\right]_{\mathrm{RD} 2} \\
& =\left[\delta_{\phi}^{(\delta \phi)}-\frac{3}{4} \delta_{\gamma_{\chi}}^{(\delta \phi)}\right]_{\phi \mathrm{D}, \mathrm{RD} 1} .
\end{aligned}
$$

With this relation, in particular, we obtain

$\Psi_{\mathrm{RD} 2}^{(\delta \phi)}=-\frac{2}{9} S_{\phi \chi}^{(\delta \phi)}$.

Density fluctuations of various components are also parameterized by $S_{\phi \chi}$. Detailed properties of the density fluctuations, however, depend on how the various components in the universe are produced. If a component $X$ is generated from the decay product of $\phi$, then there is no entropy between the photon (i.e., $\gamma_{\phi}$ ) and $X$. On the contrary, if some other scalar field $\psi$ generates $X$, the entropy between the photon and $X$ is the same as $S_{\phi \chi}$. Thus, if all the components in the universe are generated from $\phi$, the density fluctuations become purely adiabatic and

$$
\begin{aligned}
{\left[\delta_{\gamma}^{(\delta \phi)}\right]_{\mathrm{RD} 2} } & =\frac{4}{3}\left[\delta_{b}^{(\delta \phi)}\right]_{\mathrm{RD} 2} \\
& =\frac{4}{3}\left[\delta_{c}^{(\delta \phi)}\right]_{\mathrm{RD} 2} \\
& =-2 \Psi_{\mathrm{RD} 2}^{(\delta \phi)},
\end{aligned}
$$

where the subscripts $\gamma, b$, and $c$ are for the photon, baryon, and CDM, respectively. In this case, the isocurvature perturbation in the $\phi$ field is converted to the purely adiabatic density perturbation after the decay of $\phi$. On the contrary, if the baryon asymmetry is generated by the scalar field $\psi$, the entropy between the radiation and the baryon becomes $S_{\phi \chi}^{(\delta \phi)}$ and hence 511 .

$$
\begin{aligned}
& {\left[\delta_{\gamma}^{(\delta \phi)}\right]_{\mathrm{RD} 2}=\frac{4}{3}\left[\delta_{c}^{(\delta \phi)}\right]_{\mathrm{RD} 2}=-2 \Psi_{\mathrm{RD} 2}^{(\delta \phi)}} \\
& {\left[\delta_{b}^{(\delta \phi)}\right]_{\mathrm{RD} 2}=\frac{3}{4}\left[\delta_{\gamma}^{(\delta \phi)}\right]_{\mathrm{RD} 2}+\frac{9}{2} \Psi_{\mathrm{RD} 2}^{(\delta \phi)}}
\end{aligned}
$$

and in the case where $\psi$ is responsible for the $\mathrm{CDM}$ while the baryon number is somehow generated from the decay product of $\phi$,

$$
\begin{aligned}
& {\left[\delta_{\gamma}^{(\delta \phi)}\right]_{\mathrm{RD} 2}=\frac{4}{3}\left[\delta_{b}^{(\delta \phi)}\right]_{\mathrm{RD} 2}=-2 \Psi_{\mathrm{RD} 2}^{(\delta \phi)}} \\
& {\left[\delta_{c}^{(\delta \phi)}\right]_{\mathrm{RD} 2}=\frac{3}{4}\left[\delta_{\gamma}^{(\delta \phi)}\right]_{\mathrm{RD} 2}+\frac{9}{2} \Psi_{\mathrm{RD} 2}^{(\delta \phi)}}
\end{aligned}
$$

In addition, if the baryon and the CDM are both generated from sources other than $\phi$, we obtain

$$
\begin{aligned}
{\left[\delta_{\gamma}^{(\delta \phi)}\right]_{\mathrm{RD} 2} } & =-2 \Psi_{\mathrm{RD} 2}^{(\delta \phi)}, \\
{\left[\delta_{b}^{(\delta \phi)}\right]_{\mathrm{RD} 2} } & =\left[\delta_{c}^{(\delta \phi)}\right]_{\mathrm{RD} 2} \\
& =\frac{3}{4}\left[\delta_{\gamma}^{(\delta \phi)}\right]_{\mathrm{RD} 2}+\frac{9}{2} \Psi_{\mathrm{RD} 2}^{(\delta \phi)} .
\end{aligned}
$$

It is important to notice that, for the cases given in Eqs. (3.19) - (3.23), the isocurvature perturbation is correlated with the adiabatic perturbation.

So far, we have seen that the primordial fluctuation of the curvaton field may generate the metric and density fluctuations. Before closing this section, we should compare the size of the curvaton contribution with the inflaton contribution. Even with the curvaton, there is also a contribution from the inflaton fluctuation since I assume inflation as a solution to the horizon and flatness problems. If we consider the situation where there is no entropy fluctuation, inflaton contribution to the metric perturbation (in the RD2 epoch) is given by 12 .

$\Psi_{\mathrm{RD} 2}^{(\mathrm{inf})}(k)=\frac{2}{3}\left[\frac{3 H_{\mathrm{inf}}^{2}}{V_{\mathrm{inf}}^{\prime}} \times \frac{H_{\mathrm{inf}}}{2 \pi}\right]_{k=a H}$, 
with $V_{\text {inf }}^{\prime} \equiv \partial V_{\text {inf }} / \partial \chi$, while the curvaton contribution is

$\Psi_{\mathrm{RD} 2}^{(\delta \phi)}(k)=-\frac{4}{9}\left[\frac{1}{\phi_{\text {init }}} \times \frac{H_{\mathrm{inf}}}{2 \pi}\right]_{k=a H}$.

(Here and hereafter, the superscript "(inf)" is for quantities generated from the inflaton fluctuation.) As one can see, the curvaton contribution to $\Psi$ is inversely proportional to $\phi_{\text {init }}$ and hence, if $\phi_{\text {init }}$ is small enough, curvaton contribution dominates over the inflaton contribution.

Thus, in the curvaton scenario, there are two conflicting requirements on the initial amplitude of the curvaton field. One is the upper bound on $\phi_{\text {init }}$; upper bound on $\phi_{\text {init }}$ for $\Psi^{(\delta \phi)} \gtrsim \Psi^{(\mathrm{inf})}$ depends on the model of the inflation. For the case of the chaotic inflation, for example, the curvaton contribution becomes larger than the inflaton contribution if $\phi_{\text {init }} \lesssim M_{*}$. The other is the lower bound on the $\phi_{\text {init }}$, since the $\phi \mathrm{D}$ epoch cannot be realized if $\phi_{\text {init }}$ is too small. Lower bound depends on the reheating temperatures $T_{\mathrm{R} 1}$ and $T_{\mathrm{R} 2}$ given in Eqs. (2.2) and (2.3), respectively. In Fig. II I plot the lower bound on $\phi_{\text {init }}$ to realize the $\phi \mathrm{D}$ epoch.

\section{CMB angular power spectrum}

Now, I discuss the CMB angular power spectrum in the curvaton scenario. One of the most important consequences of the curvaton scenario is that, if all the components in the universe are generated only from the decay products of $\phi$, no entropy fluctuation is generated and the primordial density fluctuations (after the RD2 epoch) becomes purely adiabatic. Let us first consider such a case.

One important check point is that the scale dependence of the primordial density fluctuations. In the case where the cosmic density fluctuations are generated from the primordial fluctuation of the inflaton, scale-dependence originates from the change of the slope of the inflaton potential as well as the expansion rate during the inflation. As can be seen from Eq. (3.25), on the contrary, scale-dependence of the curvaton contribution is only from the change of the expansion rate (as far as $m_{\phi} \ll H_{\mathrm{inf}}$ ). Thus, even

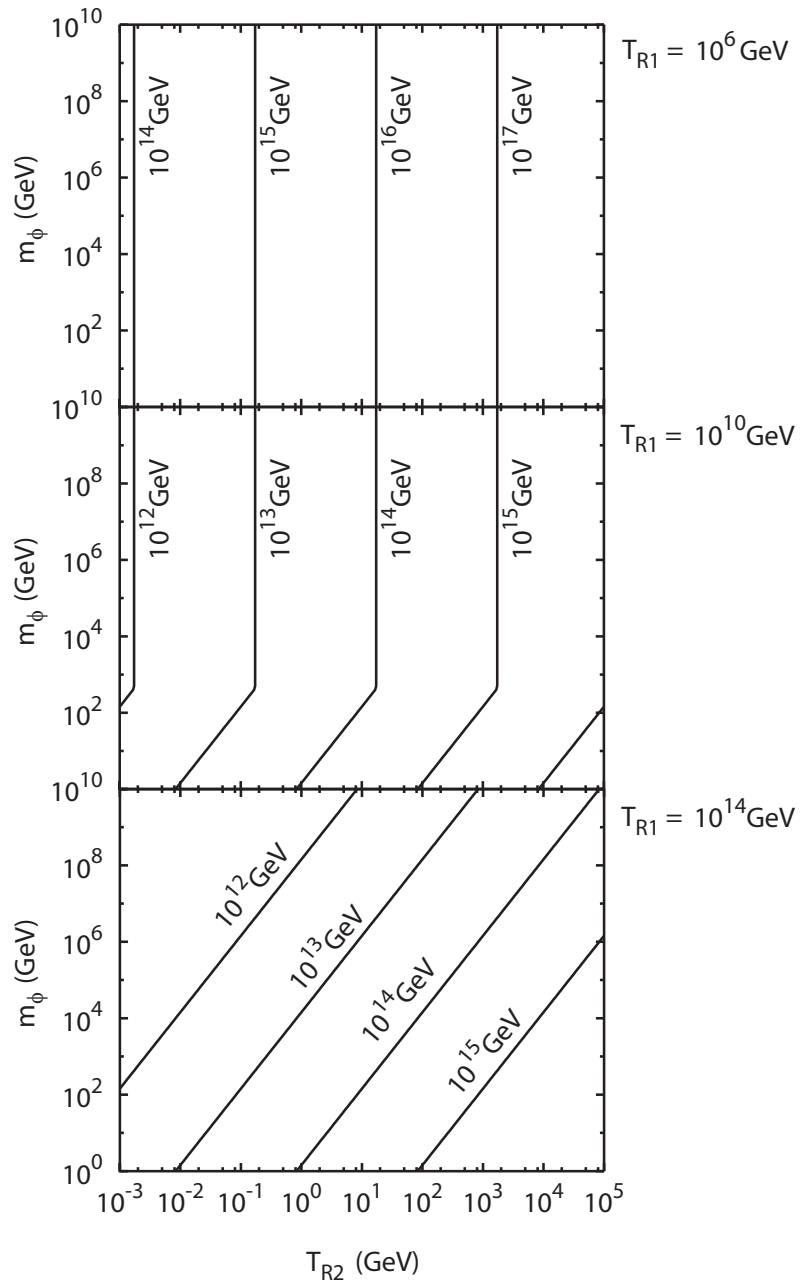

Figure 1. Lower bound on $\phi_{\text {init }}$ to realize the $\phi \mathrm{D}$ epoch on $T_{\mathrm{R} 2}$ vs. $m_{\phi}$ plane. $T_{\mathrm{R} 1}$ is taken to be $10^{6} \mathrm{GeV}, 10^{10} \mathrm{GeV}$ and $10^{14} \mathrm{GeV}$ from the top. 
though both the curvaton and inflation contributions are from primordial fluctuations of some scalar fields, their scale-dependences are different. Defining the spectral index $n_{\mathrm{S}}$ as

$\frac{d}{d \ln k} \ln \left[\Psi_{\mathrm{RD} 2}^{(\mathrm{inf}, \delta \phi)}\right]^{2} \simeq n_{\mathrm{s}}^{(\mathrm{inf}, \delta \phi)}-1$,

the spectral indices for the inflaton and curvaton contributions are calculated as

$n_{\mathrm{s}}^{(\mathrm{inf})}=1-6 \epsilon+2 \eta$,

$n_{\mathrm{s}}^{(\delta \phi)}=1-2 \epsilon$,

where $\epsilon$ and $\eta$ are slow-roll parameters which are given by

$\epsilon=\frac{1}{2} M_{*}^{2}\left(\frac{V_{\mathrm{inf}}^{\prime}}{V_{\mathrm{inf}}}\right)^{2}, \quad \eta=M_{*}^{2} \frac{V_{\mathrm{inf}}^{\prime \prime}}{V_{\mathrm{inf}}}$.

Due to the change of the scale-dependence, we can see that the observational constraints on inflation models are relaxed in the curvaton scenario. For example, for the case of the chaotic inflation with the inflaton potential $V_{\text {inf }} \propto \chi^{p_{\text {inf }}}$, spectral indices for the inflaton and curvaton contributions are estimated as $n_{\mathrm{s}}^{(\mathrm{inf})} \simeq 0.96$ and $n_{\mathrm{s}}^{(\delta \phi)} \simeq 0.98$ for $p_{\text {inf }}=2, n_{\mathrm{s}}^{(\mathrm{inf})} \simeq 0.95$ and $n_{\mathrm{s}}^{(\delta \phi)} \simeq 0.97$ for $p_{\text {inf }}=4$, and $n_{\mathrm{s}}^{(\text {inf })} \simeq 0.94$ and $n_{\mathrm{s}}^{(\delta \phi)} \simeq 0.95$ for $p_{\text {inf }}=6$. Thus, using currently available constraint on the spectral index $n_{\mathrm{s}}=$ $0.99 \pm 0.0413$ from the Wilkinson Microwave Anisotropy Probe (WMAP), simple chaotic inflation model with $p_{\text {inf }}=6$ is excluded by the observations while, with the curvaton mechanism, $p_{\text {inf }}=6$ becomes consistent with the observational constraints. For other class of inflation models, change of the constraint may be more drastic.

Next, let us discuss the effects of the possible entropy fluctuation in the curvaton scenario. As mentioned in the previous section, in the curvaton scenario, correlated mixture of the adiabatic and isocurvature fluctuations may be generated. In order to discuss effects of the entropy fluctuation to the CMB angular power spectrum, it is convenient to parameterize the density fluctuation of the non-relativistic matter as

$$
\left[\delta_{m}^{(\delta \phi)}\right]_{\mathrm{RD} 2} \equiv\left[\frac{\Omega_{b}}{\Omega_{m}} \delta_{b}^{(\delta \phi)}+\frac{\Omega_{c}}{\Omega_{m}} \delta_{c}^{(\delta \phi)}\right]_{\mathrm{RD} 2}
$$

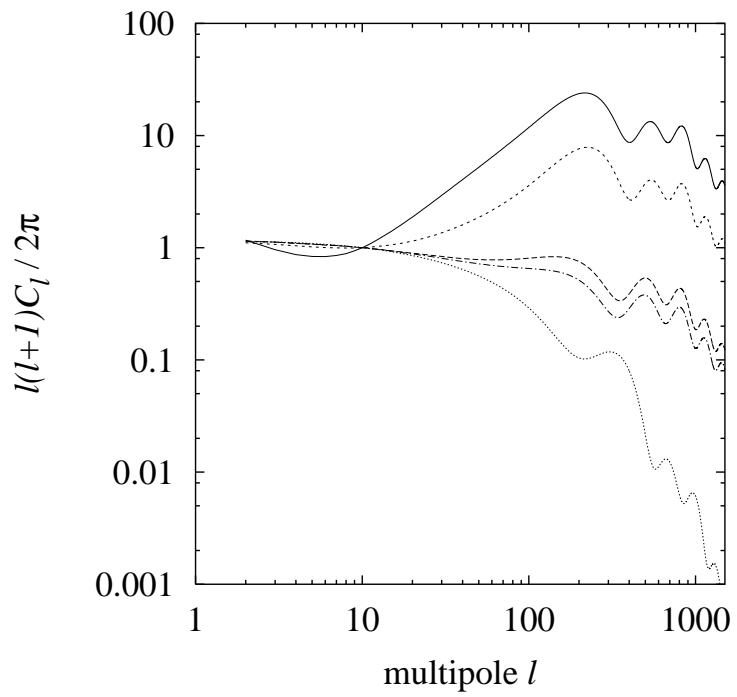

Figure 2. The angular power spectrum with correlated mixture of the adiabatic and isocurvature perturbations in the baryonic sector (solid line), in the CDM sector (long-dashed line), and in the baryonic and CDM sectors (dot-dashed line). (See Eqs. (3.19), (3.21), and (3.23), respectively.) I also show the CMB angular power spectrum for the purely adiabatic (i.e., $\kappa_{m}=0$ ) and purely isocurvature $\left(\kappa_{m}=\infty\right)$ cases with short-dashed and dotted lines, respectively. The overall normalizations are taken as $\left[l(l+1) C_{l} / 2 \pi\right]_{l=10}=1$.

$$
=\frac{3}{4}\left[\delta_{\gamma}^{(\delta \phi)}\right]_{\mathrm{RD} 2}+\kappa_{m} \Psi_{\mathrm{RD} 2}^{(\delta \phi)},
$$

where $\Omega_{b}, \Omega_{c}$ and $\Omega_{m}$ are the (present) density parameters of the baryon, the CDM, and the nonrelativistic component (and hence $\Omega_{m}=\Omega_{b}+$ $\Omega_{c}$ ). Although the density fluctuations for the baryon and the CDM are independent, shape of the CMB angular power spectrum is determined once $\kappa_{m}$ is fixed. For the purely adiabatic case, $\kappa_{m}=0$. With the relations (3.19), (3.21), and (3.23), $\kappa_{m}$ becomes $\frac{9}{2}\left(\Omega_{b} / \Omega_{m}\right), \frac{9}{2}\left(\Omega_{c} / \Omega_{m}\right)$, and $\frac{9}{2}$, respectively.

In Fig. 2] I show how the CMB angular power 
spectrum depends on the $\kappa_{m}$ parameter. As one can see, $C_{l}$ strongly depends on $\kappa_{m}$ and, if $\left|\kappa_{m}\right| \gtrsim O(0.1)$, deviation of the CMB angular power spectrum from the adiabatic result (i.e., $C_{l}$ with $\kappa_{m}=0$ ) becomes sizable. Importantly, the WMAP results strongly suggest that the primordial density fluctuations be (almost) purely adiabatic; with the WMAP data, $\kappa_{m}<0.1$ is obtained [14]. Thus, the WMAP results impose stringent constraint on the curvaton scenario. A possible small contamination of the entropy fluctuation in some case will be discussed in the next section.

\section{Implications to sneutrino cosmology}

Now, I would like to discuss the case where the curvaton field is also responsible for the scenario of baryogenesis. Among various scenarios, there are some cases where the baryon asymmetry of the universe is generated from scalar-field condensations. In those scenarios, scalar fields often dominate the universe at some epoch and hence, if they have primordial fluctuations, they may play the role of the curvaton. Probably, two of the most famous examples of the possible scalar fields responsible for the baryon asymmetry are right-handed sneutrino 1516] and the AffleckDine field [17. $\# 7$

Here, I consider the case where the righthanded sneutrino, which becomes the origin of the baryon asymmetry of the universe [15], plays the role of the curvaton [19. First, I briefly summarize the model and the thermal history. Here, the relevant part of the superpotential is given by

$W=\hat{h}_{N, i \alpha} N_{i} L_{\alpha} H_{u}+\frac{1}{2} \hat{M}_{N, i j} N_{i} N_{j}$,

where $\hat{h}_{N}$ is the Yukawa matrix for the neutrino while $\hat{M}_{N}$ is the Majorana mass matrix for the right-handed (s)neutrinos. Here, $i$ and $j$ are generation indices of the right-handed neutrino $N$ while $\alpha$ is that of the left-handed lepton doublet $L$. In addition, $H_{u}$ is the up-type Higgs

\footnotetext{
${ }^{\# 7}$ In some case, inflaton can be responsible for the scenario of the baryogenesis. For example, right-handed neutrinos can be produced by the decay of the inflaton, which may become the origin of the baryon asymmetry of the universe 18 .
}

field. I work in the basis where the matrix $\hat{M}_{N}$ is diagonalized. For simplicity, let us consider the case where the lightest right-handed sneutrino $\tilde{N}=\tilde{N}_{1}$ has non-vanishing initial amplitude. (Hereafter, mass of $\tilde{N}$ is denoted as $M_{N}$.)

With a non-vanishing primordial amplitude, $\tilde{N}$ starts to oscillate when $H \sim M_{N}$ and decays when $H \sim \Gamma_{N}$, where $\Gamma_{N}$ is the decay rate of $\tilde{N}$. Since the Majorana mass term breaks the lepton-number symmetry, lepton-number asymmetry may be generated at the time of the sneutrino decay if non-vanishing $\mathrm{CP}$ violation exists. Such a lepton-number asymmetry becomes the source of the baryon-number asymmetry of the universe with the sphaleron process.

The mechanism of generating the baryonnumber asymmetry is basically the supersymmetric version of the Fukugita-Yanagida mechanism [20]. Expression for the resultant amount of the baryon asymmetry is, however, rather complicated since, in this case, the primordial abundance of the right-handed sneutrino has nonthermally determined. Assuming $\Gamma_{N}<\Gamma_{\chi}$, we obtain 16]

$$
\begin{aligned}
\frac{n_{B}}{s} \simeq & 0.24 \times 10^{-10} \\
& \times f_{\gamma_{\tilde{N}}} \delta_{\text {eff }}\left(\frac{T_{N}}{10^{6} \mathrm{GeV}}\right)\left(\frac{m_{\nu_{3}}}{0.05 \mathrm{eV}}\right),
\end{aligned}
$$

where $T_{N}$ is the temperature at the epoch of the decay of $\tilde{N}, m_{\nu_{3}}$ the mass of the heaviest (lefthanded) neutrino mass. (So, if $\tilde{N}$ decays after dominating the universe, $T_{N}$ becomes the reheat temperature due to the decay of $\tilde{N}$.) In addition, in the basis where the Majorana mass matrix for the right-handed neutrinos $\hat{M}$ is real and diagonalized, the effective $\mathrm{CP}$ violating phase is given by

$\delta_{\mathrm{eff}}=\frac{\left\langle H_{u}\right\rangle^{2}}{m_{\nu_{3}}} \frac{\operatorname{Im}\left[\hat{h} \hat{h}^{\dagger} \hat{M}^{-1} \hat{h}^{*} \hat{h}^{T}\right]_{11}}{\left[\hat{h} \hat{h}^{\dagger}\right]_{11}}$.

Notice that, with maximum $\mathrm{CP}$ violation, $\delta_{\mathrm{eff}} \sim$ 1. In addition, $f_{\gamma}$ is the energy fraction of the radiation generated from the decay product of $\tilde{N}$. With the relation $\Gamma_{N}<\Gamma_{\chi}, \tilde{N}$ decays after the inflaton decay. In this case, it is convenient to 
define the following quantity:

$\tilde{N}_{\text {eq }} \sim\left\{\begin{array}{lll}\left(\Gamma_{N} / M_{N}\right)^{1 / 4} M_{*} & : & M_{N}<\Gamma_{\chi} \\ \left(\Gamma_{N} / \Gamma_{\chi}\right)^{1 / 4} M_{*} & : & M_{N}>\Gamma_{\chi}\end{array}\right.$.

If $\tilde{N}_{\text {init }} \sim \tilde{N}_{\text {eq }}, \rho_{\gamma_{\chi}} \sim \rho_{\tilde{N}}$ is realized when $H \sim$ $\Gamma_{\tilde{N}}$. Thus, when $\tilde{N}_{\text {init }} \lesssim \tilde{N}_{\text {eq }}, \tilde{N}$ decays in the $\gamma_{\chi}$-dominated universe and hence

$f_{\gamma_{\tilde{N}}} \sim \frac{\left(\tilde{N}_{\text {init }} / \tilde{N}_{\text {eq }}\right)^{2}}{1+\left(\tilde{N}_{\text {init }} / \tilde{N}_{\text {eq }}\right)^{2}}: \tilde{N}_{\text {init }} \lesssim \tilde{N}_{\text {eq }}$

On the contrary, if $\tilde{N}_{\text {init }} \gtrsim \tilde{N}_{\text {eq }}$, the right-handed sneutrino decays after it dominates the universe and we obtain

$f_{\gamma_{\tilde{N}}} \sim \frac{\left(\tilde{N}_{\text {init }} / \tilde{N}_{\text {eq }}\right)^{8 / 3}}{1+\left(\tilde{N}_{\text {init }} / \tilde{N}_{\text {eq }}\right)^{8 / 3}}: \tilde{N}_{\text {eq }} \lesssim \tilde{N}_{\text {init }}$.

Thus, as is easily seen, energy fraction of $\gamma_{\tilde{N}}$ becomes close to 1 when $\tilde{N}_{\text {init }} \gg \tilde{N}_{\text {eq }}$ while, in the opposite limit, most of the radiation are generated from the decay product of the inflaton.

Now, I consider the cosmological density fluctuations in this scenario. In particular, I concentrate on the case where the condensation of the right-handed sneutrino plays the role of the curvaton. (Thus, in this case, the epoch after the decay of $\tilde{N}$ is identified as the RD2 epoch.) The scenario is basically the same as the curvaton scenario which I have explained before.

In the previous discussion, I have discussed the cases where all the components in the universe are generated from the decay product of the curvaton, which corresponds to the case of $f_{\gamma_{\tilde{N}}} \simeq 1$. As is explained above, however, (small) contamination of the radiation from the decay product of the inflaton may always exist. If the baryon asymmetry is generated from thermally produced particles after the RD2 epoch is realized, then entropy fluctuations vanish and the primordial density fluctuations become purely adiabatic.

In the scenario we consider, however, the situation is quite different since the baryon asymmetry is generated from the decay product of $\tilde{N}$. In particular, if the energy fraction of the radiation from the decay product of the inflaton becomes sizable, non-vanishing baryonic entropy fluctuation may be generated.
Evolution of the density fluctuations in this case is also understood by solving the Einstein and Boltzmann equations for the fluctuations given in the previous section. We can calculate the entropy in the radiation-dominated universe after the decay of both $\chi$ and $\tilde{N}$. In the radiationdominated universe 9

$$
\begin{aligned}
\Delta_{\mathrm{tot}} & =O\left(k^{2} \tau^{2}\right), \\
\delta_{\mathrm{tot}} & =-2 \Psi_{\mathrm{RD} 2}, \\
V_{\mathrm{tot}} & =\frac{1}{2} \Psi_{\mathrm{RD} 2} k \tau .
\end{aligned}
$$

Then, with the relation

$\Delta_{\text {tot }}=f_{\gamma_{\chi}} \Delta_{\gamma_{\chi}}+f_{\gamma_{\tilde{N}}} \Delta_{\gamma_{\tilde{N}}}$,

with $\Delta_{\gamma_{X}}=\delta_{\gamma_{X}}+4\left(a^{\prime} / a\right) V_{\text {tot }} / k$, we obtain

$\Delta_{\gamma_{\tilde{N}}}^{(\delta \tilde{N})}=-\frac{f_{\gamma_{\chi}}}{f_{\gamma_{\tilde{N}}}} \Delta_{\gamma_{\chi}}^{(\delta \tilde{N})}=-6 \frac{f_{\gamma_{\chi}}}{f_{\gamma_{\tilde{N}}}} \Psi_{\mathrm{RD} 2}^{(\delta \tilde{N})}$.

Using the fact that the entropy between any component produced from $\tilde{N}$ and that from the inflaton field is conserved, we can relate $\Psi_{\mathrm{RD} 2}^{(\delta \tilde{N})}$ with $S_{\tilde{N} \chi}^{(\delta \tilde{N})}$; with the relation $S_{\tilde{N} \chi}^{(\delta \tilde{N})}=\frac{3}{4}\left(\Delta_{\gamma_{\tilde{N}}}^{(\delta \tilde{N})}-\right.$ $\left.\Delta_{\gamma_{\chi}}^{(\delta \tilde{N})}\right)$, we obtain

$\Psi_{\mathrm{RD} 2}^{(\delta \tilde{N})}=-\frac{2}{9} f_{\gamma_{\tilde{N}}} S_{\tilde{N} \chi}^{(\delta \tilde{N})}=-\frac{4}{9} f_{\gamma_{\tilde{N}}} \frac{\delta \tilde{N}_{\text {init }}}{\tilde{N}_{\text {init }}}$.

Thus, if $\tilde{N}$ decays much after it dominates the universe, $f_{\gamma_{\tilde{N}}} \simeq 1$ and hence the metric perturbation becomes comparable to the primordial entropy perturbation. On the other hand, if $f_{\gamma_{\tilde{N}}} \ll 1$, the metric perturbation becomes negligibly small. Since the baryon asymmetry is generated from $\tilde{N}$, the density fluctuation in the baryonic component is given by

$\Delta_{b}^{(\delta \tilde{N})}=\frac{3}{4} \Delta_{\gamma_{\tilde{N}}}^{(\delta \tilde{N})}=-\frac{9}{2} \frac{f_{\gamma_{\chi}}}{f_{\gamma_{\tilde{N}}}} \Psi_{\mathrm{RD} 2}^{(\delta \tilde{N})}$.

Thus, the entropy between the baryon and the radiation is given by

$$
\begin{aligned}
S_{b \gamma}^{(\delta \tilde{N})} & =\Delta_{b}^{(\delta \tilde{N})}-\frac{3}{4} \Delta_{\mathrm{tot}}^{(\delta \tilde{N})} \\
& =-\frac{9}{2} \frac{f_{\gamma_{\chi}}}{f_{\gamma_{\tilde{N}}}} \Psi_{\mathrm{RD} 2}^{(\delta \tilde{N})} \\
& =-\frac{9\left(1-f_{\gamma_{\tilde{N}}}\right)}{2 f_{\gamma_{\tilde{N}}}} \Psi_{\mathrm{RD} 2}^{(\delta \tilde{N})},
\end{aligned}
$$


and defining

$$
\kappa_{b}^{(\delta \tilde{N})} \equiv \frac{S_{b \gamma}^{(\delta \tilde{N})}}{\Psi_{\mathrm{RD} 2}^{(\delta \tilde{N})}},
$$

we obtain

$\kappa_{b}^{(\delta \tilde{N})}=-\frac{9\left(1-f_{\gamma_{\tilde{N}}}\right)}{2 f_{\gamma_{\tilde{N}}}}$.

If the right-handed sneutrino once dominates the universe, $f_{\gamma_{\tilde{N}}} \rightarrow 1$ and hence the perturbation becomes adiabatic. (Thus, the situation is like the case discussed in the previous section.)

It is interesting if a sizable amount of the energy density of the radiation is from the inflaton. In this case, $f_{\gamma_{\tilde{N}}}$ becomes smaller than 1 and correlated mixture of the adiabatic and isocurvature fluctuations is generated. As mentioned in the previous section, WMAP data suggests that the baryonic and CDM entropy fluctuations should be very small; $\left|\kappa_{b}\right|<0.5$ [14]. If $f_{\gamma_{\tilde{N}}} \sim 0.1$, however, $\left|\kappa_{b}\right|$ can be smaller than the present upper bound but the deviation of the CMB angular power spectrum from the adiabatic result may still be within the reach of the future precise observations. Thus, it is desirable to find the signal from the (correlated) entropy fluctuation in the future precise observations of the CMB anisotropy for the test of the curvaton scenario.

Finally, let me comment on the case where the curvaton also generates the baryon asymmetry of the universe via Affleck-Dine mechanism. If the primordial fluctuation of the Affleck-Dine field becomes the dominant source of the cosmological density fluctuations, large baryonic entropy fluctuation is generated even if the universe is once completely dominated by the Affleck-Dine field. This is due to the fact that the Affleck-Dine field is a complex scalar field and also that the resultant baryon asymmetry of the universe is sensitive to the initial value of the Affleck-Dine field. If the Affleck-Dine field acquires primordial fluctuation, its initial phase as well as the initial amplitude is expected to have fluctuations.

\section{Conclusions and discussion}

Here, I have discussed the basic features of the curvaton scenario and its implications to particle cosmology. In particular, I have discussed that the cosmic density fluctuations can dominantly originate from the primordial fluctuation of the "curvaton" field, which is a late-decaying scalar condensation other than the inflaton. In the curvaton scenario, scale-dependence of the cosmic density fluctuation becomes different from the case of the simple inflation, and in many cases, the spectral index $n_{\mathrm{s}}$ becomes close to 1 compared to the simple inflation case. Thus, given the fact that the CMB anisotropy observed by the WMAP suggests (almost) scale-invariant primordial density fluctuations, observational constraints on the inflaton potential can be relaxed by adopting the curvaton scenario.

One of the important feature of the curvaton scenario is the possible contamination of the (correlated) entropy fluctuation in the non-relativistic matter. The CMB angular power spectrum is sensitive to the entropy fluctuation. Thus, if such correlated entropy fluctuation is sizable, it affects the shape of the CMB angular power spectrum and hence it will be interesting and important to try to find its consequence in particular in the CMB anisotropy at future precision observations of the universe.

\section{Acknowledgments}

The author is grateful to the organizers of the Fujihara Seminar "SEESAW 1979 - 2004: Neutrino Mass and Seesaw Mechanism" for their hospitality during the stay. The work of T.M. is supported by the Grant-in-Aid for Scientific Research from the Ministry of Education, Science, Sports, and Culture of Japan, No. 15540247.

\section{REFERENCES}

1. A. H. Guth, Phys. Rev. D 23 (1981) 347; K. Sato, MNRAS 195 (1981) 467.

2. S. Kasuya, T. Moroi and F. Takahashi, arXiv:hep-ph/0312094

3. K. Enqvist and M. S. Sloth, Nucl. Phys. B 626 (2002) 395.

4. D. H. Lyth and D. Wands, Phys. Lett. B $\mathbf{5 2 4}$ (2002) 5 . 
5. T. Moroi and T. Takahashi, Phys. Lett. B 522 (2001) 215 [Erratum-ibid. B 539 (2002) 303].

6. N. Bartolo and A. R. Liddle, Phys. Rev. D 65 (2002) 121301; T. Moroi and T. Takahashi, Phys. Rev. D 66 (2002) 063501; D. H. Lyth, C. Ungarelli and D. Wands, Phys. Rev. D 67 (2003) 023503; M. S. Sloth, Nucl. Phys. B 656 (2003) 239; R. Hofmann, arXiv:hep-ph/0208267 K. Dimopoulos and D. H. Lyth, arXiv:hep-ph/0209180 T. Moroi and H. Murayama, Phys. Lett. B 553 (2003) 126; K. Enqvist, S. Kasuya and A. Mazumdar, Phys. Rev. Lett. 90 (2003) 091302; F. Di Marco, F. Finelli and R. Brandenberger, Phys. Rev. D 67 (2003) 063512; K. A. Malik, D. Wands and C. Ungarelli, Phys. Rev. D 67 (2003) 063516; M. Postma, Phys. Rev. D 67 (2003) 063518; C. Gordon and A. Lewis, arXiv:astro-ph/0212248 K. Dimopoulos, Phys. Rev. D 68 (2003) 123506; A. R. Liddle and L. A. Urena-Lopez, Phys. Rev. D 68 (2003) 043517; J. McDonald, Phys. Rev. D 68 (2003) 043505; K. Dimopoulos, G. Lazarides, D. Lyth and R. Ruiz de Austri, JHEP 0305 (2003) 057; K. Enqvist, A. Jokinen, S. Kasuya and A. Mazumdar, Phys. Rev. D 68 (2003) 103507; K. Dimopoulos, D. H. Lyth, A. Notari and A. Riotto, JHEP 0307 (2003) 053 M. Endo, M. Kawasaki and T. Moroi, Phys. Lett. B 569 (2003) 73; M. Postma and A. Mazumdar, arXiv:hep-ph/0304246 M. Postma, arXiv:astro-ph/0305101 S. Kasuya, M. Kawasaki and F. Takahashi, Phys. Lett. B 578 (2004) 259; D. H. Lyth and D. Wands, Phys. Rev. D 68 (2003) 103515; D. H. Lyth and D. Wands, Phys. Rev. D 68 (2003) 103516; K. Dimopoulos, G. Lazarides, D. Lyth and R. R. de Austri, Phys. Rev. D 68 (2003) 123515; K. Hamaguchi, M. Kawasaki, T. Moroi and F. Takahashi, arXiv:hep-ph/0308174 D. H. Lyth, Phys. Lett. B 579 (2004) 239; N. Bartolo, S. Matarrese and A. Riotto, Phys. Rev. D 69 (2004) 043503; J. McDonald, JCAP 0312 (2003) 005; J. McDonald, arXiv:hep-ph/0310126 C. Gordon and K. A. Malik, arXiv:astro-ph/0311102 M. Bastero-Gil, V. Di Clemente and
S. F. King, arXiv:hep-ph/0311237 S. Gupta, K. A. Malik and D. Wands, arXiv:astro-ph/0311562 R. Allahverdi and M. Drees, arXiv:hep-ph/0401054 L. Pilo, A. Riotto and A. Zaffaroni, arXiv:astro-ph/0401302 E. J. Chun, K. Dimopoulos and D. Lyth, arXiv:hep-ph/0402059 M. Postma, arXiv:astro-ph/0403213 D. Langlois and F. Vernizzi, arXiv:astro-ph/0403258 K. Enqvist, arXiv:hep-ph/0403273 J. McDonald, arXiv:hep-ph/0404154 M. Ikegami and T. Moroi, arXiv:hep-ph/0404253.

7. G. Veneziano, Phys. Lett. B 265 (1991) 287; M. Gasperini and G. Veneziano, Astropart. Phys. 1 (1993) 317; M. Gasperini and G. Veneziano, Phys. Rev. D 50 (1994) 2519; M. Gasperini and G. Veneziano, Phys. Rep. 373 (2003) 1.

8. J. Khoury, B. A. Ovrut, P. J. Steinhardt and N. Turok, Phys. Rev. D 64 (2001) 123522; J. Khoury, B. A. Ovrut, N. Seiberg, P. J. Steinhardt and N. Turok, Phys. Rev. D 65 (2002) 086007; J. Khoury, B. A. Ovrut, P. J. Steinhardt and N. Turok, Phys. Rev. D 66 (2002) 046005.

9. W. T. Hu, Ph. D Thesis (arXiv:astro-ph/9508126).

10. S. Mollerach, Phys. Rev. D 42 (1990) 313.

11. T. Moroi and T. Takahashi, in [6].

12. J. M. Bardeen, P. J. Steinhardt and M. S. Turner, Phys. Rev. D 28 (1983) 679.

13. D. N. Spergel et al., Astrophys. J. Suppl. 148 (2003) 175.

14. K. Hamaguchi, M. Kawasaki, T. Moroi and F. Takahashi, in Ref. 6].

15. H. Murayama, H. Suzuki, T. Yanagida and J. Yokoyama, Phys. Rev. Lett. 70 (1993) 1912.

16. K. Hamaguchi, H. Murayama and T. Yanagida, Phys. Rev. D 65 (2002) 043512.

17. I. Affleck and M. Dine, Nucl. Phys. B 249 (1985) 361.

18. T. Asaka, K. Hamaguchi, M. Kawasaki and T. Yanagida, Phys. Rev. D 61 (2000) 083512. 19. T. Moroi and H. Murayama, in 6].

20. M. Fukugita and T. Yanagida, Phys. Lett. B 
174 (1986) 45. 